\title{
VACUUM ENERGY IN GAUGED EXTENDED SUPERGRAVITY
}

\author{
B. ALLEN and S. DAVIS \\ DAMTP, Unversity of Cambridge, Silver Street, Cambrige CB3 9EW, UK
}

Recelved 4 I ebruary 1983

\begin{abstract}
The one loop vacuum energy in gauged extended supergravity is calculated in antı de Sitter space using zeta function regularisation. It vanishes for $N \geqslant 5$. The energy zeta function has no poles for $N \geqslant 3$.
\end{abstract}

It has been shown [1] that antı de Sitter spacetime, with cosmological constant $\Lambda=-3 e^{2} / k^{2}$, is the ground state of gauged extended supergravity theories [2]. Here $e$ is the gauge coupling constant and $\kappa^{2} / 4 \pi=G /$ $\hbar c$ is Newton's gravitational constant. The cosmological constant may be changed by the vacuum energy of the fields. In flat space, the total vacuum energy is zero, because the boson and fermion contributions cancel exactly, to all orders in perturbation theory [3]. However, in antı de Sitter space, no such termby-term cancellation occurs, because the bosons and fermions have different energy spectra. In this paper, we show a simple way to calculate the resulting vacuum energy, at one loop.

Since the anti de Sitter covering space is not compact, the spectrum of the hamiltonian is continuous. However, after imposing a simple energy conservation condition at spatial infinity [2], a discrete spectrum is obtained. Antı de Sitter space may be regarded as a potentral energy well, surrounded by a reflecting wall. By permitting no energy flux through this wall, the resulting states are bound, and possess a discrete energy spectrum [4]. The supersymmetry transformations can then be used to show that the energy levels are discrete for the higher-spin fields as well.

The energy eigenstates of a field of helicity $L$ and spin $|L|$ are labeled by a radial quantum number $n$ and angular momentum quantum numbers $(j, m)$. The allowed values of $L$ and 0 (scalar), $\pm 1 / 2$ (spinor), \pm 1 (vector), $\pm 3 / 2$ (gravitino) and \pm 2 (graviton) As usual in the case of spherical symmetry, we have $n=0,1,2$, 3 ,.. and $\jmath=|L|,|L|+1,|L|+2$, . and $m=-\jmath,-\jmath+1$,
. , $J-1, j$. If a boson state $(n, J, m)(L$ integer $)$ contains $p$ quanta, then its contribution to the total energy will be

$E=\left(p+\frac{1}{2}\right) E(n, j), \quad p=0,1,2,3$.

If a fermion state $(n, j, m)$ ( $L$ half-integer) contains $p$ quanta, then its contribution to the total energy will be

$E=\left(p-\frac{1}{2}\right) E(n, j), \quad p=0,1$.

Here, the energy of each quanta in the state $(n, j, m)$ is [2] $E(n, j)=(n+j+1) \hbar \omega$ where $\hbar \omega=e c^{2} / \kappa$ $=c^{2}\left[-\frac{1}{3} \Lambda\right]^{1 / 2}$. Because the energies do not depend on $m$, the sum over all states $\Sigma_{n, j, m}$ will always be written as $\Sigma_{n, j}(2 j+1)$.

The vacuum has no quanta present, and so $p=0$ in (1) and (2). We could then formally write the total vacuum energy for a supermultiplet as the sum over all boson and fermion states

$E_{\mathrm{vac}}=\frac{1}{2}\left(\sum_{\mathrm{B}} E(n, j)-\sum_{\mathrm{F}} E(n, j)\right)$.

However, it is readily apparent from (1) and (2) that both of these sums are divergent. Moreover, we can group the terms so that their difference (3) converges to any desired value. Consequently, a regularisation procedure needs to be adopted.

A simple method is to form the generalised zeta function [5] from the energy eigenvalues for the field with helicity $L$, 


$$
\begin{aligned}
& \zeta_{L}(z)=\sum_{n, j, m}[E(n, j)]^{-z} \\
& =(\hbar \omega)^{-z} \sum_{n=0}^{\infty} \sum_{j=|L|}^{\infty}(2 j+1)(n+j+1)^{-z} .
\end{aligned}
$$

This sum converges on the half-plane $\operatorname{Re}(z)>3$ and can be analytically continued to a meromorphic function with poles only at $z=1$ and $z=3$. Its value at $z$ $=-1$ is finite, and $\frac{1}{2} \zeta_{L}(-1)$ represents the vacuum energy of a spin $|L|$ field. Then the zeta function for a supermultiplet is

$\zeta_{\text {multiplet }}(z)$

$$
\begin{aligned}
& =\left(\sum_{L \text { integer }}-\sum_{L \text { half-1nteger }}\right) d(L) \zeta_{L}(z) \\
& =\sum_{L}(-1)^{2 L} d(L) \zeta_{L}(z),
\end{aligned}
$$

where $d(L)$ is the number of helicity $L$ fields in the multiplet, given in table 1 . The value of $\zeta_{\text {multiplet }}$ at $z=-1$

$E_{\text {vac }}=\frac{1}{2} \zeta_{\text {multiplet }}(-1)$,

is the total vacuum energy of all the fields.

The zeta function (4) for a spin $|L|$ field may be evaluated by grouping the terms according to the value of $n+j$. For the moment, we drop the absolute value sign from $L$,

$$
\begin{aligned}
& \zeta_{L}(z)=(\hbar \omega)^{-z} \\
& \quad \times \sum_{n=0}^{\infty} \sum_{j=0}^{\infty}(2 J+2 L+1)(n+j+L+1)^{-z} \\
& =(\hbar \omega)^{-z}\left[(2 L+1)(L+1)^{-z}\right. \\
& \left.+[(2 L+1)+(2 L+3)](L+2)^{-z}+\right] \\
& =(\hbar \omega)^{-z} \sum_{k=1}^{\infty}\left[2 k L+k^{2}\right](k+L)^{-z} \\
& =(\hbar \omega)^{-z}\left(\sum_{k=1}^{\infty}(k+L)^{-z+2}-L^{2} \sum_{k=1}^{\infty}(k+L)^{-z}\right), \\
& \zeta_{L}(z)=(\hbar \omega)^{-z}\left[\zeta(z-2,|L|+1)-L^{2} \zeta(z,|L|+1)\right] .
\end{aligned}
$$

The generalised Riemann zeta function $\zeta(z, \alpha)$ is defined for $\operatorname{Re}(z)>1$ by

\begin{tabular}{|c|c|c|c|c|c|c|c|c|c|c|c|}
\hline \multirow[t]{2}{*}{$N$} & \multicolumn{9}{|l|}{$L$} & \multirow[b]{2}{*}{$E_{\text {vac }}$} & \multirow[b]{2}{*}{$R_{1}$} \\
\hline & 2 & $\frac{3}{2}$ & 1 & $\frac{1}{2}$ & 0 & $-\frac{1}{2}$ & -1 & $-\frac{3}{2}$ & -2 & & \\
\hline 1 & 1 & 1 & & & & & & & & $\frac{469}{384} \hbar \omega$ & $-\frac{7}{4}(\hbar \omega)^{-1}$ \\
\hline 2 & 1 & 2 & 1 & & & & & & & $\frac{157}{192} \hbar \omega$ & $-\frac{1}{2}(\hbar \omega)^{-1}$ \\
\hline 3 & 1 & 3 & 3 & 1 & & & & & & $\frac{15}{32} \hbar \omega$ & \\
\hline 4 & 1 & 4 & 6 & 4 & 1 & & & & & $\frac{3}{16} \hbar \omega$ & \\
\hline 5 & 1 & 5 & 10 & 10 & 5 & 1 & & & & & \\
\hline 6 & 1 & 6 & 15 & 20 & 15 & 6 & 1 & & & & \\
\hline 7 & 1 & 7 & 21 & 35 & 35 & 21 & 7 & 1 & & & \\
\hline 8 & 1 & 8 & 28 & 56 & 70 & 56 & 28 & 8 & 1 & & \\
\hline
\end{tabular}

$\zeta(z, \alpha)=\sum_{k=0}^{\infty}(k+\alpha)^{-z}$

and by analytic continuation elsewhere [6] .

The function $\zeta(z, \alpha)$ is meromorphic in $z$ except for a simple pole at $z=1$ with residue 1 . It reduces to the ordinary Riemann zeta function $\zeta(z)$ when $\alpha=1$. If $z$ is a nonpositive integer, it is

Table 1

The left-hand side of this table gives $d(L)$, the number of particle states of helicity $L$ in a theory with $N$ supersymmetry generators. The right-hand side lists the vacuum energy in these theories, and the residue of the pole at $z=1$ of $\zeta$ multiplet $(z)$. 
$\zeta(-n, \alpha)=-B_{n+1}(\alpha) /(n+1), \quad n=0,1,2,3$

where $B_{n}(\alpha)$ are Bernoullı's polynomials

The vacuum energy of a supermultiplet can now be obtained. Using the formulae

$B_{2}(\alpha)=\alpha^{2}-\alpha+\frac{1}{6}$,

$B_{4}(\alpha)=\alpha^{4}-2 \alpha^{3}+\alpha^{2}-\frac{1}{30}$,

we find from (7) and (9) that

$\zeta_{L}(-1)=\hbar \omega\left(\frac{1}{4} L^{4}-\frac{1}{6} L^{2}+\frac{1}{120}\right)$.

Then from (5) and (6)

$$
\begin{aligned}
E_{\mathrm{vac}} & =\frac{1}{2} \sum_{L}(-1)^{2 L} d(L) \zeta_{L}(-1) \\
= & \frac{\hbar \omega}{2} \sum_{L}(-1)^{2 L} d(L)\left(\frac{1}{4} L^{4}-\frac{1}{6} L^{2}+\frac{1}{120}\right) .
\end{aligned}
$$

The reader can easily verify the spin sum rules of ref. [7]. For a theory with $N$ supersymmetry generators $(1 \leqslant N \leqslant 8)$ there are $N$ spin sum rules. They state that

$\sum_{L}(-1)^{2 L} d(L) L^{P}=0$

$$
P \text { integer , } 0 \leqslant P \leqslant N-1,
$$

which implies that all contributions on the rhs of (12) vanish for $N \geqslant 5$. Thus $E_{\mathrm{vac}}=0$. Shown in table 1 are the nonzero values of $E_{\mathrm{vac}}$ for $N<5$. In the flatspace limit $\Lambda \rightarrow 0$, they also vanish.

Because the only pole in $\zeta(z, \alpha)$ is at $z=1$, we can write

$\zeta_{L}(z)=\frac{r_{1}(L)}{z-1}+\frac{r_{3}(L)}{z-3}+f_{L}(z)$,

where $f_{L}(z)$ is an entire function of $z$. Then from (7), the residues at $z=1$ and $z=3$ are

$r_{1}(L)=-(\hbar \omega)^{-1} L^{2}, \quad r_{3}(L)=(\hbar \omega)^{-3}$.

The poles in the zeta functions for different spins may cancel when summed (5) to form $\zeta_{\text {multiplet }}(z)$. The residue of the poles at $z=1$ and $z=3$ are

$$
\begin{aligned}
R_{1} & =\sum_{L}(-1)^{2 L} d(L) r_{1}(L) \\
& =-(\hbar \omega)^{-1} \sum_{L} i(-1)^{2 L} d(L) L^{2}, \\
R_{3} & =\sum_{L}(-1)^{2 L} d(L) r_{3}(L) \\
& =(\hbar \omega)^{-3} \sum_{L}(-1)^{2 L} d(L) .
\end{aligned}
$$

The spin sum rules (13) now imply that $\zeta_{\text {multiplet }}(z)$ has no pole at $z=3$ for any $N$ and no pole at $z=1$ for $N \geqslant 3$. The residue at $z=1$ is given in table 1 for $N$ $<3$.

The appearance of poles in a four-dimensional zeta function corresponds to an infinite renormalisation of the gravitational and cosmological constants [5]. We believe they have a similar interpretation for the three. dimensional energy zeta function.

The result that there is no cosmological constant renormalisation at one loop for $N \geqslant 5$ has been shown by very different arguments [8-10]. This may hold to all orders in perturbation theory [11]. In our paper, the one loop cancellation has been explicitly demonstrated in antı de Sitter space.

Because the supersymmetric ground state has zero energy, it follows that supersymmetry is broken for $N<5$. What does it break to" Perhaps its ground state has nonzero temperature and angular momentum in antı de Sitter space. By calculating the many-particle partition function from (1) and (2), the properties of this ground state may be determined.

We would like to thank G.W. Gibbons and S.W. Hawking for helpful discussions. B A. is grateful for a research scholarship from the Marshall Aid Commemoration Commission and S.D. for a research scholarship from Trinity College.

\section{References}

[1] B de Wit and N. Nicola1, Nucl Phys B188 (1981) 98, Phys. Lett. B108 (1981) 285.

[2] P. Breitenlohner and D.Z Freedman, Phys. Lett. B115 (1982) 197, Ann. Phys 144 (1982) 249;

G.W Gibbons, C.M. Hull and N.P Warner, The stability of gauged supergravity, to be published in Nucl. Phys B (1983). 
[3] B. Zumino, Nucl. Phys. B89 (1975) 535.

[4] S.J. Avis, C.J. Isham and D. Storey, Phys. Rev. D18 (1978) 3565.

[5] S.W. Hawking, Commun. Math Phys. 55 (1977) 133.

[6] W. Magnus, F. Oberhettınger and R.P. Sonı, Formulae and theorems for the special functions of mathematical physics, 3rd Ld. (Springer, Berlın, 1966).

[7] T.L. Curtwright, Phys. Lett. 102B (1981) 17.

[8] M.J. Duff, in Supergravity '81, eds. S. Ferrara and J G. Taylor (Cambridge U P., Cambridge, 1982).
[9] M.J. Duff, in Quantum Gravity 2, eds. C.J. Isham, R. Penrose and D.W. Scrama (Oxford U.P., Oxford, 1981)

[10] S.M. Christensen, M.J. Duff, G.W. Gibbons and M Rocek, One-loop effects in supergravity with a cosmological constant, University of Cambridge DAMTP preprint (1981), unpublished, Phys. Rev Lett. 45 (1980) 161.

[11] K S. Stelle and P.K. Townsend, Phys. Lett 113B (1982) 25. 